\title{
The color evolution of the optical afterglow of GRB 030329 and the implications for the underlying supernova SN 2003dh
}

\author{
V. Šimon ${ }^{1}$, R. Hudec ${ }^{1}$, and G. Pizzichini ${ }^{2}$ \\ 1 Astronomical Institute, Academy of Sciences of the Czech Republic, 25165 Ondřejov, Czech Republic \\ e-mail: simon@asu.cas.cz \\ 2 IASF/CNR, Sezione di Bologna, via Gobetti 101, 40129 Bologna, Italy
}

Received 4 February 2004 / Accepted 30 June 2004

\begin{abstract}
We find that the color indices $(B-V)_{0},(V-R)_{0},(R-I)_{0},(I-J)_{0}$ in the observer frame of the optical afterglow (OA) of GRB 030329 during $t-T_{0}<10$ days are consistent with those of a uniform group of 25 OAs of GRBs, previously analyzed by Šimon et al. $(2001 ; 2003 a)$. The synchrotron component with parameters similar to those of the other 25 OAs was thus initially dominant in the optical and IR spectrum of the OA of GRB 030329. Following the arguments of Šimon et al. (2001), it also appears that either GRB 030329 was not located deep inside a star-forming region or that the local dust was destroyed by the intense initial flash. The absolute $R$ magnitude of this event $M_{R_{0}} \approx-26.6 \mathrm{mag}$ in the rest frame at $\left(t-T_{0}\right)_{\text {rest }}=0.25$ days is in good agreement with the typical brightness of the OAs analyzed by Šimon et al. (2001). We detected large color variations of the OA of GRB 030329 only for $t-T_{0}>12$ days, which suggest a rapid evolution of the spectrum of the underlying SN 2003dh. We compare it with the individual type Ic SNe and find that its color evolution is plausibly matched only by that of SN 1998bw although the indices still reveal spectral differences between the two objects. The agreement is particularly good only at $t-T_{0}=26$ and 27 days. The evolution of SN 2003dh becomes faster than that of SN 1998bw later on and SN 2003dh develops an excess light in the red/IR spectral region. We also emphasize the discordance with other type Ic SNe which are not associated with GRBs.
\end{abstract}

Key words. gamma rays: bursts - radiation mechanisms: non-thermal - ISM: jets and outflows - galaxies: ISM stars: supernovae: general - stars: supernovae: individual: SN 2003dh

\section{Introduction}

Color indices have been and still are widely used in the study of many phenomena in astrophysics. Even quite recently the use of broad-band colors has been advocated for identification of young type Ib/c SN (Gal-Yam et al. 2004). For the study of optical afterglows (OAs) of GRBs this powerful method is only very rarely applied, therefore in this field it is still quite innovative. Color indices enable us to resolve small variations of the profile of the spectra of the OAs from the measurements in the commonly used $U B V R I J$ filters. This method proved to be very useful for the analysis of the common properties of the OAs (Šimon et al. 2001 and 2003a). It also allows us to constrain the properties of the local interstellar medium of GRBs inside their host galaxies.

The long, intense gamma-ray burst GRB 030329 with the duration of $50 \mathrm{~s}$ was discovered by HETE-II on March 29, 2003 (Vanderspek et al. 2003). An exceptionally bright OA $(\sim 13 \mathrm{mag}(R))$ was discovered at $t-T_{0} \approx 1.25 \mathrm{~h}$ (Peterson $\&$ Price 2003; Price et al. 2003), which enabled investigators to promptly determine the redshift $z=0.168$ (Greiner et al. 2003a; Hjorth et al. 2003) and to follow the light evolution to very late phases. The light curve of the OA declined with an initial mean decay rate with $\alpha=-1.115$ (Uemura et al. 2003). The features of the type Ic SN 2003dh appeared in the spectrum after about a week and strengthened later on (Stanek et al. 2003; Hjorth et al. 2003; Matheson et al. 2003; Lipkin et al. 2004). The spectrum displayed large similarities to another type Ic SN 1998bw, associated with GRB 980425 (Galama et al. 1998).

In this letter, we apply the method of color indices to the OA of GRB 030329 and show that it enables the separation of the contributions of the individual emission mechanism, and the tracing of the evolution of SN 2003dh in later phases of the event. We also show that this event has characteristics quite similar to the ensemble of OAs analyzed by Šimon et al. (2001 and 2003a). A preliminary version of this analysis was presented by Šimon et al. (2003b).

\section{Sources of the data and data analysis}

The bulk of the data used for this analysis comes from the papers by Lipkin et al. (2004) and Matheson et al. (2003). These observations were supplemented by the data provided by 


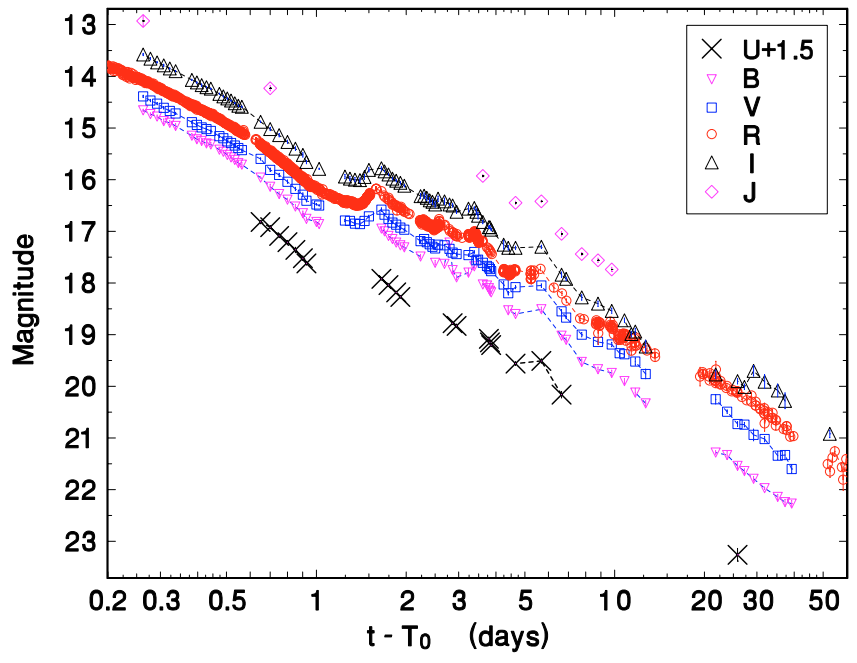

Fig. 1. The light curves of the OA of GRB 030329 in the individual passbands. The whole $R$ band light curve is displayed to show the time evolution of the brightness while for other filters only those (usually averaged) points which were used for the calculation of the color indices are plotted. Error bars are included but they are usually smaller than the symbols used. In densely covered intervals the points are connected by lines for convenience. (This figure is available in color in electronic form.)

Lamb et al. (2003), Zharikov et al. (2003a,b), Ibrahimov et al. (2003a,b), Bikmaev et al. (2003), Gorosabel et al. (2003).

The light curves with the dense coverage (BVRI) were binned into the segments of 0.015 days in the early phase of the OA ( $t-T_{0}<0.6$ days) while the bins of 0.025 days were used for 0.6 days $<t-T_{0}<0.95$ days. The very densely covered $R$ band light curve served as an indicator of the rapid changes on the time scale of hours in the early phase of the OA. This enabled us to choose the intervals free of rapid changes; a reliable interpolation between the neighbouring measurements in the segments or filters where the coverage was sparser (within less than an hour in the first few days after the GRB, and within a night in the late phases) was thus possible. For later times, a centroid was often calculated from a group of closely spaced data (less than 1.5-2 $\mathrm{h}$ apart for 1.6 days $<t-T_{0}<13$ days; less than one day apart for $t-T_{0}>21$ days) in a given filter - this enabled us to achieve a higher accuracy of the brightness. The reliability of the measurements from the GCN Circulars was checked both as regards their quoted standard deviations and their agreement with the observations of Lipkin et al. (2004) and Matheson et al. (2003). We find that the data do not need any further photometric calibration. For some time intervals it was not possible to determine the colors because of the lack of suitable data. Data in Fig. 1 are not normalized, because any fitting of the data will give rise to the different parameters of the fits for the measurements in the individual filters, which will completely distort the color indices. In general, color indices are never determined from the normalized data. Our approach is described in Šimon et al. (2001). We note that throughout this paper $t-T_{0}$ are given for $z=0.168$, unless otherwise stated.

The standard deviations of the indices were calculated from the quoted errors of the data in each filter in the case of a single point or from the standard deviations of the means in the case of a centroid or a bin. The resulting standard deviations of the indices lie within 0.02-0.14 mag and are displayed in the figures. The Galactic reddening toward GRB 030329 is only $E_{B-V}=0.02$ mag according to Schlegel et al. (1998), and smaller than the standard deviations of the indices, it therefore could be neglected for our purposes. Also any light contribution of the host galaxy was quite small (upper limit $R \approx 23.1 \mathrm{mag}$ ) (Blake \& Bloom 2003) and could be neglected. Indeed, there is no flattening in the late phase of the resulting light curves (Fig. 1) which could be attributed to the contribution of the host galaxy. The color indices throughout this paper are in the observer frame to allow a comparison with the papers by Šimon et al. (2001 and 2003a).

The time evolution of the color indices during the whole event is displayed in Fig. 2. For comparison, also the mean colors with the standard deviation of the ensemble of 25 OAs $\left(t-T_{0}<10\right.$ days) (Šimon et al. 2003a), are shown. They were recalculated, here without including the OA of GRB 030329, but this hardly altered them. The OAs of the following GRBs were used: 970228, 970508, 971214, 980519, 980613, 980703, 990123, 990308, 990510, 990712, 991208, 991216, 000301C, 000911, 000926, 010222, 010921, 011121, 011211, 020405, 020813, 021004, 021211, 030226, 030418. We note that the analyses by Šimon et al. (2001 and 2003a) were restricted to $t-T_{0}<10$ days due to practical reasons, since for most OAs the data for later epochs are sparse, with large uncertainties and mostly only in the $R$ filter. The detail of the dramatic color changes during the late phase of the OA of GRB 030329 can be seen in Fig. 3. The rapid large reddening occurs first in $B-V$, then $V-R$, and finally in $R-I$. The well defined profiles of the time evolution of the indices also imply that the paths in the color-color diagrams (Figs. 4 and 5) are real.

We generated synthetic color indices of SN 1998bw using the code at http: //wise-obs . tau.ac.il/ dovip/typing (Poznanski et al. 2002), with both the wavelengths of the passbands and $t-T_{0}$ scaled to $z=0.168$, with $T_{0}$ referring to the time of GRB 980425. The maximum of brightness of SN 1998bw occurred at $t-T_{0} \approx 18$ days for $z=0.168$. The indices of SN 1998bw inside a similar interval of $t-T_{0}$ as that covered for the OA of GRB 030329 are included in Figs. 2, 4 and 5. We also generated the colors of type Ic SN 2002ap and the group of type Ic SNe in the same way, and their time evolution is shown in Fig. 2. In these cases, the time scales were recalculated taking the rise time to the maximum brightness $\sim 10$ days at $z \approx 0$ (Mazzali et al. 2002). Any small uncertainty in the rise time scale will not influence our results. Since only the color evolution of SN 1998bw yields a plausible match to SN 2003dh, the other SNe are not shown in Figs. 4 and 5 to avoid overcrowding.

The mean color indices of the OA of GRB 030329 for $t-T_{0}<10$ days were determined to be $(B-V)_{0}=0.34 \pm 0.08$, $(V-R)_{0}=0.35 \pm 0.04,(R-I)_{0}=0.47 \pm 0.04,(I-J)_{0}=$ $0.82 \pm 0.07$. Although some variations of the indices occur during this time interval, they are considerably smaller than in later epochs; the calculation of the mean color index is thus meaningful for $t-T_{0}<10$ days. These mean colors enable a comparison with the colors of other OAs (Šimon et al. 2001, see also below). 

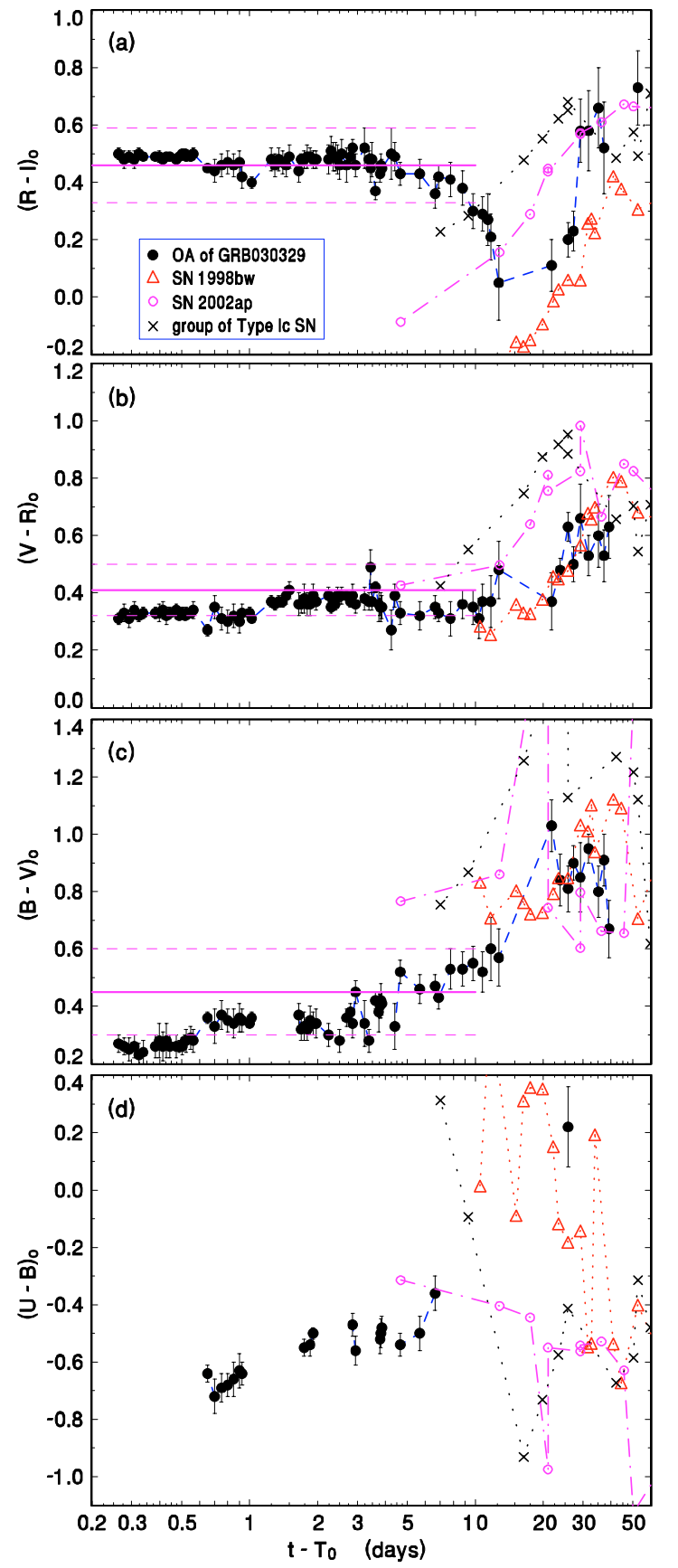

Fig. 2. The time evolution of the color indices of the OA of GRB 030329 (solid circles). The points are connected by the lines in the densely covered parts of the curves for convenience. The standard deviation of each point is included. The horizontal solid line with the dashed error bars marks the mean color indices of the ensemble of 25 OAs, determined by Šimon et al. (2003a). The synthetic colors of SN 1998bw, SN 2002ap and the group of type Ic SNe, with the passbands and $t-T_{0}$ recalculated for $z=0.168$, appropriate for GRB 030329, are also plotted for comparison. The colors of the $25 \mathrm{OAs}$ are found to be independent of the redshift. (This figure is available in color in electronic form.)

\section{Discussion}

We found that the $(B-V)_{0},(V-R)_{0},(R-I)_{0}$ and $(I-J)_{0}$ colors of the OA of GRB $030329(z=0.168)$ in the observer frame and

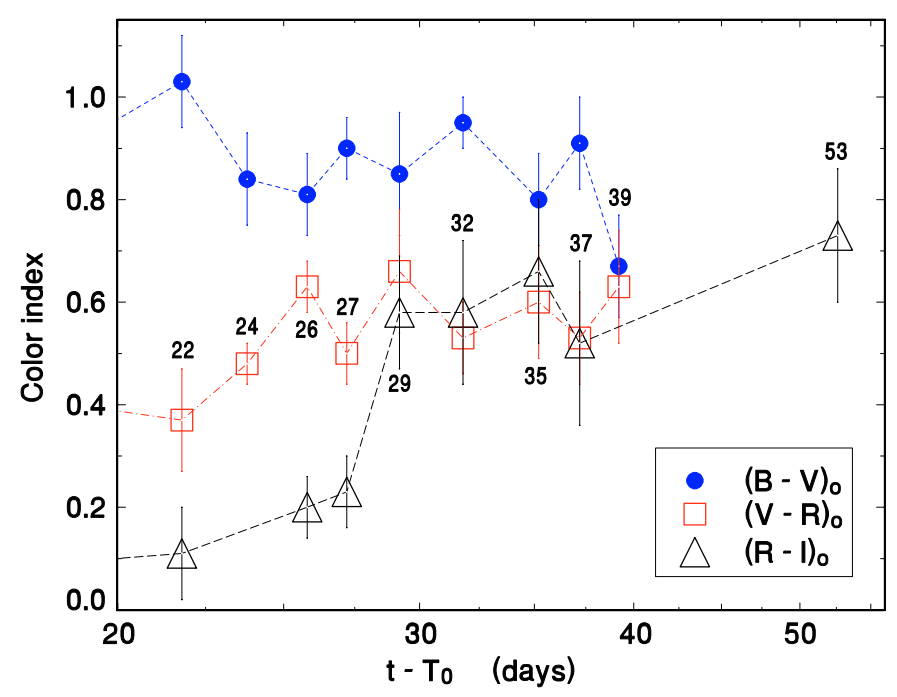

Fig. 3. Color changes of the OA of GRB 030329 during the late phase. The points are connected by the line to clearly trace the time evolution of the colors. Notice that the reddening occurs first in $B-V$, then $V-R$, and finally in $R-I$. The numbers at the points denote $t-T_{0}$ in days to allow an easy comparison with Figs. 4 and 5. (This figure is available in color in electronic form.)

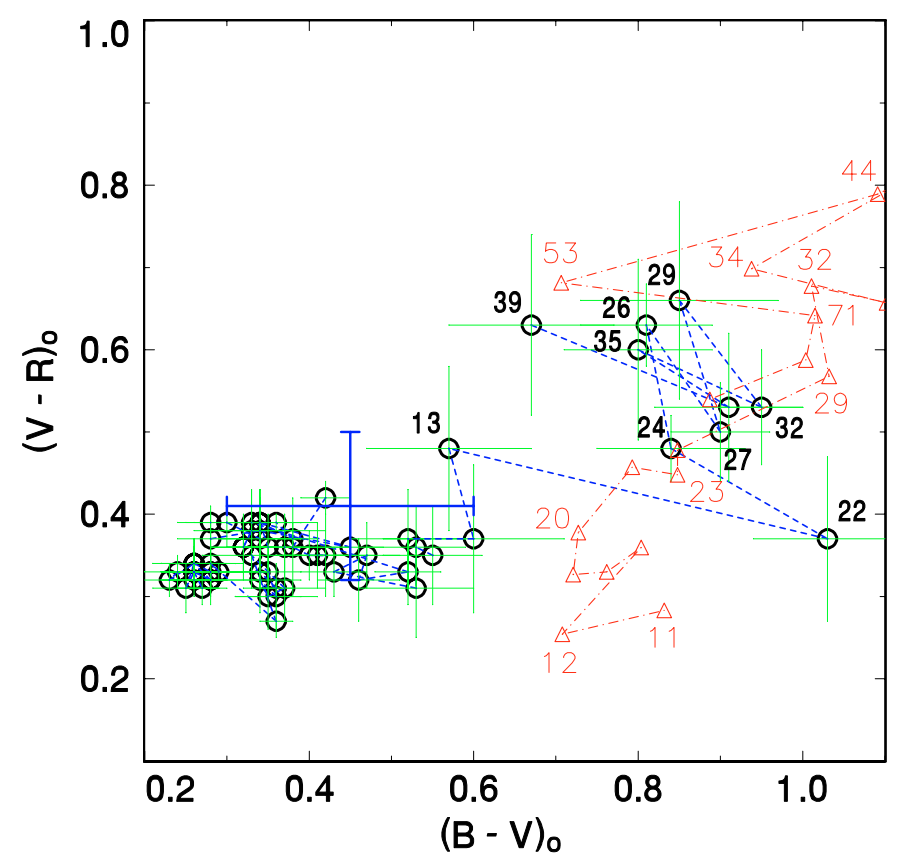

Fig. 4. $B-V$ vs. $V-R$ diagram of the OA of GRB 030329 (empty circles). The points are connected by the line to show the time evolution of the colors. The large cross denotes the centroid and standard deviations of the colors of the ensemble of 25 OAs for $t-T_{0}<10$ days (Šimon et al. 2003a). Empty triangles mark the synthetic colors of SN 1998bw. The numbers denote $t-T_{0}$ in days (for $z=0.168$ ), starting from the appropriate GRBs. (This figure is available in color in electronic form.)

their time evolution inside the interval $t-T_{0}<10$ days are in very good agreement with those of a uniform group of 25 OAs with $z=0.36-3.5$, determined by Šimon et al. (2001 and 2003a). These colors are consistent with the GRB afterglow fireball emission model by Sari et al. (1998). This suggests 


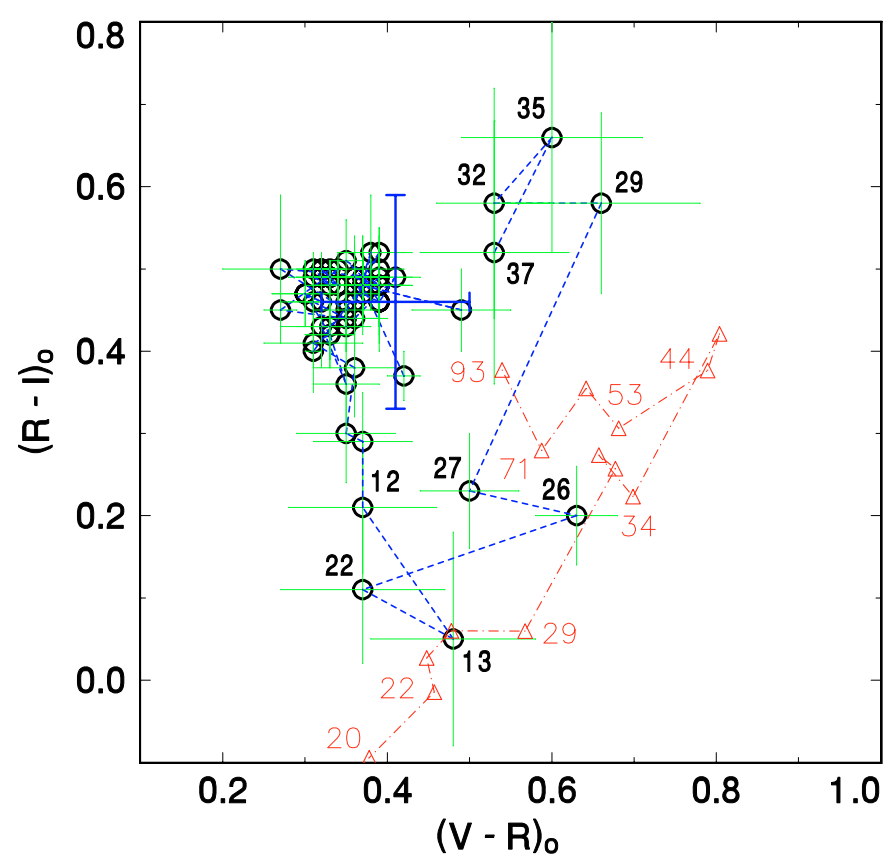

Fig. 5. $V-R$ vs. $R-I$ diagram of the OA of GRB 030329. The arrangement is analogous to Fig. 4. (This figure is available in color in electronic form.)

that the synchrotron component with parameters comparable to the other OAs was initially dominant in the spectrum of the OA of GRB 030329. Although the colors of the OA for $t-T_{0}<10$ days are still consistent with other OAs, a larger motion due to the $B-V$ variations can be seen in Fig. 4 . The standard deviation of $B-V$ of the ensemble larger than that of $V-R$ can suggest that the spectral variations increasing toward shorter wavelengths can be rather common in the OAs.

We argue that the intrinsic reddening of GRB 030329 inside its host galaxy must have been quite low $\left(E_{B-V} \leq 0.1 \mathrm{mag}\right)$ because the colors of this OA in the first 10 days are in good agreement with those of the ensemble of 25 OAs. The argument is such that in the case of a large reddening, it would be quite unlikely to obtain such similar values of absorption in all cases (see Šimon et al. 2001, for details). There are three possibilities: the GRBs with detectable OAs are observed only when they lie near the Earth-watching side of a star-forming region, or the density and dust abundance of the local interstellar medium is substantially reduced by the intense initial flash (models by Waxman \& Draine 2000), or the dust abundance in the host galaxy is very low due to the low metal abundance. The fact that GRB 030329 with its $z=0.168$ fits the colors of the other 25 OAs with various $z$ up to 3.5 (and hence presumably occurring in largely different times of the evolution of their host galaxies) speaks in favour of the first possibility. This is also supported by the moderate metallicity of the host (Matheson et al. 2003). An argument for a low reddening, based on an independent method of detecting the low polarization of the OA of GRB 030329 and its time evolution was presented also by Greiner et al. (2003b). Also they preferred the first of the three possibilities.

We detect prominent color changes of the OA of GRB 030329 only at $t-T_{0}>12$ days (Figs. 3-5), which suggest a rapid evolution of the spectrum of SN 2003dh. Changes of a large part of the optical and near IR continuum of SN 2003dh, and not only of the line(s), must be involved in the observed complicated color variations. In our case, the $R-I$ and $V-R$ indices provide us with the most reliable discrimination among the individual subtypes of type Ic SNe. It emerges that the individual type Ic SNe display a considerable diversity in both the values of the color indices at a given $t-T_{0}$ and, at some extent, also in the time evolution of the colors, particularly for $t-T_{0}<30$ days (Fig. 2). The very rapid color evolution of $R-I$ and $V-R$ of SN 2003dh (especially their rise) is plausibly matched only by that of SN 1998 bw but the indices still reveal spectral differences between the two objects. The agreement between the colors of SN 2003dh and SN 1998bw is particularly good only at $t-T_{0}=26$ and 27 days when SN 1998bw was already on its decay from the maximum light. The brightness of SN 2003dh was thus already declining, too. This finding is in agreement with the spectroscopic dating by Hjorth et al. (2003) who conclude that the explosion of SN 2003dh and GRB 030329 agreed within \pm 2 days, and with the fact that type Ic SNe reach the maximum optical brightness about 10-16 days after the onset of the explosion (Mazzali et al. 2002). It is remarkable that the evolution of SN 2003dh begins to be faster than that of SN 1998bw later on $\left(t-T_{0}>27\right.$ days), as suggested from the paths in Figs. 4 and 5. SN 2003dh also appears to develop an excess light in the red/IR spectral region in comparison with SN 1998bw, as suggested by its systematically larger $(R-I)_{0}$ for $t-T_{0}>27$ days. We also emphasize good agreement between these two SNe in $U-B$ and the large discordance with other type Ic SNe which are not known to be associated with GRBs. Figure 2 also shows that for most time considered here both SN 2003dh and SN 1998bw remain bluer than other type Ic $\mathrm{SNe}$ (except for $U-B$ ).

The absolute $R$ magnitude of the OA of GRB 030329 $M_{R_{0}}=-25.7 \mathrm{mag}$ at $\left(t-T_{0}\right)_{\text {rest }}=0.25$ days, determined according to the approach used by Šimon et al. (2001), is in good agreement with the average $M_{R_{0}}$ of the ensemble of OAs. Zero order k-correction, introduced by Šimon et al. (2001), leads to only a slightly brighter $M_{R_{0}}$ roughly $-26.6 \mathrm{mag}$. On the other hand, type Ic SNe reach $M_{\mathrm{V}}$ only between -17 and -19.4 mag at maximum (e.g. Mazzali et al. 2002). This also implies that the break in the light curve at $t-T_{0} \approx 1$ day (Fig. 1) cannot be explained by the emerging light contribution of SN 2003dh, because it occurred at a too bright absolute magnitude and with the colors not corresponding to a supernova.

In summary, for GRB 030329, an event particularly important, because it is sure to coincide with a SN, a detailed analysis by the method of color indices enables us to show that it has initial colors and absolute magnitude similar to other GRBs. It also enables us to separate the contributions of the emission mechanisms, namely synchrotron radiation and $\mathrm{SN}$, based on the commonly available multiband photometry. This approach is important also for analyses of faint OAs for which obtaining spectra with good signal to noise ratio at late phases is often impossible. We also stress that the evolution in the late phase of the OA of GRB 030329 was prominent only in the color indices, not in the light curves. The color indices are thus a much 
better tool to resolve the evolution of the supernova in the profile of an OA than the light curves themselves.

Acknowledgements. This study was supported by the grant A3003206 provided by the Grant Agency of the Academy of Sciences of the Czech Republic, and the project ESA PRODEX INTEGRAL 14527. We also acknowledge the CNR-AVČR collaborative project Investigation of GRBs (2000/2003). We thank N. Masetti for a calculation of the Galactic extinction towards GRB 030329.

\section{References}

Bikmaev, I., Khamitov, I., Sakhibullin, N., et al. 2003, GCN Circ., No. 2220

Blake, C., \& Bloom, J. S. 2003, GCN Circ., No. 2011

Galama, T. J., Vreeswijk, P. M., van Paradijs, J., et al. 1998, Nature, 395, 670

Gal-Yam, A., Poznanski, D., Maoz, D., et al. 2004, PASP, 116, 597

Gorosabel, J., de Ugarte Postigo, A., Castro-Tirado, A. J., et al. 2003, GCN Circ., No. 2242

Greiner, J., Peimbert, M., Estaban, C., et al. 2003a, GCN Circ., No. 2020

Greiner, J., Klose, S., Reinsch, K., et al. 2003b, Nature, 426, 157

Hjorth, J., Sollerman, J., Moller, P., et al. 2003, Nature, 423, 847

Ibrahimov, M. A., Asfandiyarov, I. M., Kahharov, B. B., et al. 2003a, GCN Circ., No. 2191
Ibrahimov, M. A., Asfandiyarov, I. M., Kahharov, B. B., et al. 2003b, GCN Circ., No. 2219

Lamb, D. Q., York, D., Barentine, J., et al. 2003, GCN Circ., No. 2040

Lipkin, Y. M., Ofek, E. O., Gal-Yam, A., et al. 2004, ApJ, 606, 381

Matheson, T., Garnavich, P. M., Stanek, K. Z., et al. 2003, ApJ, 599, 394

Mazzali, P. A., Deng, J., Maeda, K., et al. 2002, ApJ, 572, L61

Peterson, B. A., \& Price, P. A. 2003, GCN Circ., No. 1985

Poznanski, D., Avishay, G.-Y., Maoz, D., et al. 2002, PASP, 114, 833

Price, P. A., Fox, D. W., Kulkarni, S. R., et al. 2003, Nature, 423, 844

Sari, R., Piran, T., \& Narayan, R. 1998, ApJ, 497, L17

Schlegel, D. J., Finkbeiner, D. P., \& Davis, M. 1998, ApJ, 500, 525

Stanek, K. Z., Matheson, T., Garnavich, P. M., et al. 2003, ApJ, 591, L17

Šimon, V., Hudec, R., Pizzichini, G., \& Masetti, N. 2001, A\&A, 377, 450

Šimon, V., Hudec, R., Pizzichini, G., \& Masetti, N. 2003a, GRB Symp., AIP Conf. Proc. (in press)

Šimon, V., Hudec, R., \& Pizzichini, G. 2003b, GRB Symp., AIP Conf. Proc. (in press)

Uemura, M., Kato, T., Ishioka, R., et al. 2003, Nature, 423, 843

Vanderspek, R., Crew, G., Doty, J., et al. 2003, GCN Circ., No. 1997

Waxman, E., \& Draine, B. T. 2000, ApJ, 537, 796

Zharikov, S., Tovmassian, G., \& Richer, M. 2003a, GCN Circ., No. 2171

Zharikov, S., Chavushyan, V., \& Mujica, R. 2003b, GCN Circ., No. 2245 